\title{
EFEITO DO DESBASTE E DA FERTILIZAÇÃO NA PORCENTAGEM DE CASCA E CONICIDADE DE TORAS DE Eucalyptus grandis
}

\author{
Israel Luiz de Lima ${ }^{1}$, José Nivaldo Garcia ${ }^{2}$ \\ ${ }^{1}$ Eng. Florestal, Dr., Instituto Florestal do Estado de São Paulo, São Paulo, São Paulo, Brasil - israelluizde.lima@ yahoo.com.br \\ ${ }^{2}$ Eng. Florestal, Dr., Depto. de Ciência Florestais da ESALQ-USP, Piracicaba, São Paulo, Brasil - jngarcia@esalq.usp.br
}

Recebido para publicação: 13/08/2009 - Aceito para publicação: 26/08/2010

\begin{abstract}
Resumo
O uso de espécies do gênero Eucalyptus, como madeira serrada, já é uma realidade, porém estudos de técnicas silviculturais que podem influenciar no aproveitamento da madeira serrada tornam-se necessários. O presente trabalho teve como objetivo estudar a influência do desbaste e da fertilização na porcentagem de casca e na conicidade de toras de uma população de Eucalyptus grandis W. Hill ex Maiden, aos 21 anos de idade, em Lençóis Paulista/SP. Para tanto, foram analisadas três intensidades de desbastes seletivos (37, 50 e 75\%), presença ou ausência de fertilizantes na época de início dos desbastes, e duas classes de DAP (classe 1 para valores entre 25 e 30 e classe 2 para valores entre 30 e $35 \mathrm{~cm}$ ). As amostras, em forma de toras de $3 \mathrm{~m}$, foram coletadas em duas posições verticais ao longo da altura do fuste comercial (tora 1 de 1,7 a 4,7 e tora 2 de 6,4 a 9,4 m), tratadas de forma independente. A porcentagem de casca foi influenciada pelo desbaste e pela fertilização, porém somente na tora 1. A classe de diâmetro não exerceu nenhum efeito nessa característica da árvore. A conicidade variou somente com a classe de diâmetro, sendo maior nas toras da $2^{\mathrm{a}}$ classe.

Palavra-chave: Manejo florestal; qualidade das toras; características dendrométricas.
\end{abstract}

\begin{abstract}
Effects of thinning and fertilization on percentage of bark and log taper of Eucalyptus grandis. Genus Eucalyptus species used as sawn wood is already reality, although it is still necessary more research on silvicultural techniques that may influence sawn wood usage. This research aim to analyze influence of thinning and fertilization on percentage of bark and log taper of a planted population of Eucalyptus grandis W. Hill ex Maiden, 21 years-old, in Lençóis Paulista, SP. It focused three thinning intensities (37, 50 and 75\%), presence or absence of fertilizers and two DBH classes (class 1 for values between 25 and 30 and class 2 for values between 30 and $35 \mathrm{~cm}$ ). Samples of $3 \mathrm{~m}$. long logs had been collected from two vertical positions at the height of tree commercial stem (log 1 from 1.7 to 4.7 and $\log 2$ from 6.4 to $9.4 \mathrm{~m}$ ), and treated independently. Bark percentage was influenced by thinning intensity and fertilization, but only in $\log 1$. The percentage of bark was influenced by thinning intensity and fertilization but only in $\log 1$. Diameter class did not bring any effect on such tree characteristic. Log tapering varied only accordingly to the diameter class, and it was larger for $2^{\text {nd }}$ class logs.

Keywords: Forest management; quality of logs; dendrometric characteristics.
\end{abstract}

\section{INTRODUÇÃO}

É cada vez maior o interesse na utilização de madeira serrada de algumas espécies do gênero Eucalyptus, podendo-se destacar o Eucalyptus grandis, espécie mais cultivada no Brasil, por apresentar boas propriedades físico-mecânicas, versatilidade no seu uso, beleza e coloração natural com grande aceitação para utilização refinada em interiores.

De acordo com Lima (2005), por meio de técnicas de manejo florestal pode-se controlar a qualidade da madeira, mas, para isso, é necessário conhecimento das propriedades das madeiras e até que ponto elas são influenciadas pelas características genéticas e pelas modificações do ambiente (espaçamento, proporção de copa viva e taxa de crescimento).

O desbaste e a adubação são tratamentos silviculturais que controlam a competição entre árvores, 
influenciando os diversos parâmetros que compõem a qualidade da madeira. A conicidade e a porcentagem de casca nas toras das árvores são variáveis que podem influenciar no rendimento em madeira serrada quando do desdobro (LIMA, 2005).

Segundo Grosser (1980), a conicidade, que é a diminuição do diâmetro do tronco da base para a copa da árvore, pode ser influenciada pelo ambiente, principalmente pelo espaçamento. Na prática, essa característica é considerada um defeito quando o diâmetro da tora diminui mais de um centímetro por metro de comprimento, principalmente quando está acima dos $2 \mathrm{~m}$ de altura.

De acordo com Garcia (2002), a conicidade influencia a qualidade mecânica da madeira serrada e, principalmente, sua homogeneidade ao longo do comprimento da peça. Em sua pesquisa com toras de E. grandis e E saligna, Crêspo (2000) observou que essa característica das toras influenciou mais o rendimento em madeira serrada do que as rachaduras de extremidade de toras e de tábuas.

As árvores que são plantadas em espaçamento mais amplo normalmente crescem mais rapidamente em diâmetro do que aquelas plantadas em espaçamento mais estreito. Entretanto, em determinada idade, elas terão maior tendência ao forquilhamento, devido à ação dos ventos, maior conicidade, maior tortuosidade, ramos mais grossos e copas mais extensas que as das árvores de plantios mais densos. Essas árvores podem favorecer a formação de maior proporção de lenho de reação (COUTO et al., 1977; DANIEL et al., 1979).

De acordo com Paula Neto; Nunes (1992), o efeito tecnológico e econômico da casca em espécies de eucalipto constitui-se num problema para certos setores florestais. Assim, é importante o conhecimento da porcentagem de casca existente e a sua variação ao longo do tronco.

O presente trabalho teve como objetivo avaliar o efeito do desbaste e da fertilização na porcentagem de casca e na conicidade de toras de Eucalyptus grandis, aos 21 anos de idade, em duas classes diamétricas e duas posições verticais ao longo da altura do fuste comercial da árvore.

\section{MATERIAL E MÉTODOS}

O material utilizado nesta pesquisa foi obtido de uma população experimental de Eucalyptus grandis W. Hill ex Maiden, aos 21 anos de idade, com nível de melhoramento de P. S. C. (Pomar de Semente Clonal) e procedência Botucatu (origem Coff's Harbour), localizada no município de Lençóis Paulista/SP e pertencente à Ripasa S/A Celulose e Papel. O solo local é classificado como Latossolo Vermelho Amarelo, de textura média, e o clima é do tipo CWa, segundo a classificação de Köppen.

O ensaio foi composto de seis parcelas de $900 \mathrm{~m}^{2}$, representando cada uma um tratamento diferente. O plantio das árvores foi realizado em 16/12/1982, em espaçamento de 3,0 x 1,5 m, totalizando 2.222 árvores/ha. Inicialmente foram aplicados $222 \mathrm{~kg} / \mathrm{ha}$ de NPK 6:30:6, distribuídos nos sulcos de plantio.

Em 1987 foram iniciados desbastes do tipo seletivo, com base no número de árvore por hectare, nas intensidades de 37, 50 e 75\%. Após o desbaste, foi realizada uma adubação com $1000 \mathrm{~kg}$ de NPK 12:06:12 e $100 \mathrm{~kg}$ de lama-cal por hectare, distribuídos nas entrelinhas de apenas três das parcelas, caracterizando, portanto, tratamentos com e sem adubação. Em seguida a essa intervenção silvicultural, foi efetuada uma gradagem leve, para incorporar esses elementos ao solo. Após o último dos três desbastes, que ocorreu aos 8,5 anos, o experimento foi mantido sem alterações até o início do presente estudo. Cabe ressaltar ainda que o plantio populacional não sofreu desrama artificial. Na tabela 1 são apresentados os tratamentos da população experimental que foram selecionados para estudo.

Realizou-se inicialmente um inventário do experimento e, a partir da distribuição diamétrica obtida, elegeram-se duas classes de DAP's (diâmetro à altura do peito) para o estudo, sendo a $1^{\text {a }}$ classe composta de árvores com DAP entre 25 e $30 \mathrm{~cm}$ e a $2^{\mathrm{a}}$ classe composta de árvores com DAP entre $30 \mathrm{e}$ $35 \mathrm{~cm}$.

Dentro de cada uma das parcelas foram selecionadas oito árvores, sendo quatro em cada uma das classes definidas, totalizando 48 árvores, que foram devidamente identificadas no campo. Foram tomadas duas toras de cada uma das árvores, sendo a primeira (tora 1 ), com $3 \mathrm{~m}$ de comprimento, retirada à altura de 1,70 a $4,70 \mathrm{~m}$, e a segunda (tora 2), com $3 \mathrm{~m}$ de comprimento, na altura de 6,4 a 9,40 m. Não foram utilizadas as toras basais de $1,70 \mathrm{~m}$ de comprimento, principalmente porque elas sofrem influência do alargamento da base da árvore. 
Tabela 1. Tratamentos selecionados dentro de uma população experimental de E. grandis de 21 anos de idade em Lençóis Paulista/SP.

Table 1. Treatments selected from an experimental population of a 21 year-old grown E. grandis, in Lençóis Paulista, SP.

\begin{tabular}{lcccccc}
\hline Tratamento & $\begin{array}{c}\text { Adubação } \\
\text { aos 5 anos* }\end{array}$ & $\begin{array}{c}(\%) \\
\text { Desbaste }\end{array}$ & $\begin{array}{c}\text { Idade no 1 } \\
\text { desbaste }\end{array}$ & $\begin{array}{c}\text { Idade no 2 } \\
\text { desbaste }\end{array}$ & $\begin{array}{c}\text { Idade no 3 } \\
\text { desbaste }\end{array}$ & $\begin{array}{c}\mathbf{N}^{\mathbf{0}} \text { de árvores/ha } \\
\text { aos 21 anos }\end{array}$ \\
\hline 1 & $\mathrm{~N}$ & $37 \%$ & 5 & 6,5 & 8,5 & 549 \\
2 & $\mathrm{~S}$ & $37 \%$ & 5 & 6,5 & 8,5 & 521 \\
3 & $\mathrm{~N}$ & $50 \%$ & 5 & 7,5 & - & 535 \\
4 & $\mathrm{~S}$ & $50 \%$ & 5 & 7,5 & - & 556 \\
5 & $\mathrm{~N}$ & $75 \%$ & 5 & - & - & 535 \\
6 & $\mathrm{~S}$ & $75 \%$ & 5 & - & - & 535 \\
\hline
\end{tabular}

Fonte: Lima et al. (2007).

*N: não adubado; S: adubado.

Foram avaliadas as variáveis porcentagem de casca (PC) e conicidade (C) de cada tora. A porcentagem de casca foi obtida pela equação (1), a partir das áreas das secções transversais calculadas com os respectivos diâmetros, com e sem casca, de cada tora.

$$
P C=\left(\frac{A_{c c}-A_{s c}}{A_{c c}}\right) 100
$$

Em que: $\mathrm{PC}=$ porcentagem de casca $(\%)$;

$$
\begin{aligned}
& A_{c c}=D_{c c}^{2}+d_{c c}^{2} ; \\
& A_{s c}=D_{s c}^{2}+d_{s c}^{2} \\
& \mathrm{D}_{c c} \text { e } \mathrm{D}_{s c}=\text { diâmetros com e sem casca, obtidos na extremidade mais grossa }(\mathrm{cm}) ; \\
& \mathrm{d}_{\mathrm{cc}} \text { e } \mathrm{d}_{\mathrm{sc}}=\text { diâmetros com e sem casca, obtidos na extremidade mais fina }(\mathrm{cm}) .
\end{aligned}
$$

A conicidade de cada tora foi calculada utilizando-se a equação (2).

$$
C=\left(\frac{D_{c c}-d_{c c}}{L}\right)
$$

Em que: $\mathrm{C}=$ conicidade da tora $(\mathrm{cm} / \mathrm{m})$;

$\mathrm{L}=$ comprimento da tora $(\mathrm{m})$;

$\mathrm{D}_{\mathrm{cc}}=$ diâmetros com casca, obtidos na extremidade mais grossa $(\mathrm{cm})$;

$\mathrm{d}_{\mathrm{cc}}=$ diâmetros com e sem casca, obtidos na extremidade mais fina $(\mathrm{cm})$.

As hipóteses testadas foram: a) o desbaste, a adubação e a classe diamétrica não influenciam na conicidade e porcentagem de casca nas toras de E. grandis de 21 anos; b) não existem variações na conicidade e porcentagem de casca ao longo da altura da árvore de E. grandis.

A avaliação do experimento foi efetuada utilizando-se modelos estatísticos que foram ajustados para considerar a variação completa de todos os tratamentos, dentro de cada uma das posições da altura. Inicialmente foi efetuado o teste de homogeneidade de variância e para isso utilizou-se o teste de Hartley. Posteriormente foi feito o teste $\mathrm{F}$ de análise de variância segundo o delineamento experimental inteiramente casualizado, adotando-se o esquema fatorial 3 × 2 × 2 (desbastes x adubo x classe), para cada tora em separado.

Foi aplicado o teste de Tukey, sempre que observada diferença significativa, ao nível de 5\% de significância, de algum tratamento no teste F. Para comparar a posição da tora na altura da árvore, foi utilizado o Teste t pareado. Os resultados obtidos das variáveis foram analisados estatisticamente com o auxílio do procedimento estatístico PROC GLM do SAS (SAS, 1999), considerando-se as variáveis intensidades de desbaste e adubo como fixas e a variável classe como aleatória. 


\section{RESULTADOS E DISCUSSÃO}

Na tabela 2 encontram-se os resumos dos resultados das análises de variância para porcentagem de casca (PC).

Tabela 2. Resumo da análise de variância efetuada para a porcentagem de casca (PC) de E. grandis aos 21 anos de idade em Lençóis Paulista/SP.

Table 2. Summary of the variance analysis made for percentage of bark (PC) of a 21 year-old grown $E$. grandis, in Lençóis Paulista, SP.

\begin{tabular}{|c|c|c|c|}
\hline \multirow{2}{*}{ Causa de variação } & \multirow{2}{*}{ GL } & \multicolumn{2}{|c|}{ Quadrados médios } \\
\hline & & Tora 1 & Tora 2 \\
\hline Intensidade de desbaste (I) & 2 & $2,6495^{*}$ & $5,4261^{\mathrm{n} . \mathrm{s}}$ \\
\hline Adubo (A) & 1 & $1,4106^{*}$ & $0,8621^{\mathrm{n} . \mathrm{s}}$ \\
\hline Classe (C) & 1 & $8,1684^{\mathrm{n} . \mathrm{s}}$ & $0,0528^{\text {n.s }}$ \\
\hline (I) $\mathrm{x}(\mathrm{A})$ & 2 & $0,6321^{\mathrm{n} . \mathrm{s}}$ & $7,8128^{\text {n.s }}$ \\
\hline (I) $\mathrm{x}(\mathrm{C})$ & 2 & $0,0777^{\mathrm{n} . \mathrm{s}}$ & $2,1497^{\mathrm{n} . \mathrm{s}}$ \\
\hline (A)x(C) & 1 & $0,0052^{\text {n.s }}$ & $0,2725^{\text {n.s }}$ \\
\hline (I) $x(A) x(C)$ & 2 & $0,6694^{\mathrm{n} . \mathrm{s}}$ & $1,9202^{\mathrm{n} . \mathrm{s}}$ \\
\hline Resíduo & 36 & 2,7140 & 2,2879 \\
\hline $\mathrm{CV}_{\mathrm{e}}(\%)$ & & 20,72 & 22,70 \\
\hline Média (\%) & & 7,95 & 6,66 \\
\hline
\end{tabular}

Verifica-se, de acordo com os resultados obtidos, que ocorreram diferenças significativas entre as intensidades de desbastes, para a porcentagem de casca, apenas na tora 1. A porcentagem de casca na intensidade de $75 \%$ foi significativamente menor do que a intensidade de 50\% (Figura 1).

Para o fator adubo, foi observado que houve diferenças significativas somente para tora 1 , em que as porcentagens de casca das toras das parcelas adubadas foram significativamente maiores do que as não adubadas (Figura 1).

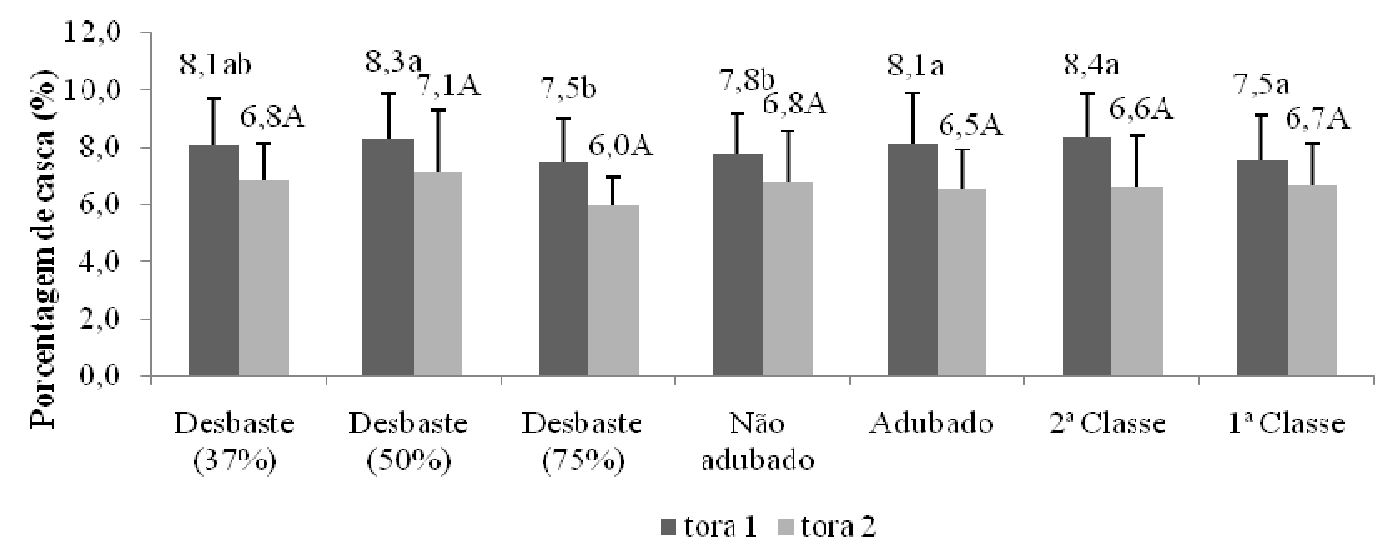

Nota: Médias seguidas da mesma letra não diferem entre si pelo teste Tukey ao nível de 5\% de significância.

Figura 1. Porcentagem de casca (PC) de E. grandis aos 21 anos de idade.

Figure 1. Percentage of bark (PC) of a 21 year-old grown E. grandis.

No fator classe de DAP, não ocorreram diferenças significativas entre toras. Os resultados demonstraram que as intensidades de desbaste e a fertilização influenciaram somente na porcentagem de casca da tora 1, podendo-se, então, considerar que a porcentagem de casca das toras retiradas em alturas menores são mais influenciadas por esses fatores e que a classe de DAP não interferiu nos resultados, para as condições deste trabalho. 
De acordo com a literatura, os valores de porcentagem de casca encontrados $(6,0$ a $8,4 \%)$ podem ser considerados baixos, interferindo, então, pouco no rendimento em madeira serrada. Os valores obtidos estão abaixo do observado por Oliveira et al. (1999) e Barchet (2001), e foram semelhantes ao obtidos por Haselein et al. (2004).

Stanger (1993) não verificou diferenças no comportamento da espessura da casca de E. dunni de 5 anos de idade, cultivadas em dois locais diferentes. A espécie em estudo apresentou pequena espessura de casca. Calori; Kikute (1997) observaram que a porcentagem de cascas de E. dunni, aos 20 anos idade, decresceu até $50 \%$ da altura comercial e cresceu a partir desse ponto até $100 \%$.

Tolfo et al. (2005) observaram em um estudo com 25 clones de Eucalyptus spp. que a porcentagem de casca apresentou alta estimativa de herdabilidade, no sentido amplo $\left(\mathrm{H}^{2}=0,75\right)$, sugerindo que essa variável sofre pequena influência ambiental.

Estudo realizado por Campos; Ribeiro (1983), com 486 árvores de Eucalyptus spp., mostrou uma boa correlação negativa entre o valor percentual de casca e a altura do tronco, entretanto ocorreu uma correlação positiva entre percentual de casca e diâmetro.

Lopes (2000) verificou, para E. saligna com 22 anos de idade, uma correlação positiva entre a massa específica básica e a porcentagem de casca, ou seja, árvores com maior massa específica apresentaram maior porcentagem de casca. $\mathrm{O}$ autor observou também uma tendência de diminuição da porcentagem de casca com a altura da árvore, mesma tendência observada por Paula Neto; Nunes (1992).

$\mathrm{Na}$ tabela 3 encontra-se o resumo dos resultados das análises de variância efetuadas para a conicidade das toras. Foi possível observar que não ocorreram diferenças significativas dessa característica, tanto para diferentes intensidades de desbaste como para o fator adubo.

Tabela 3. Resumo da análise de variância efetuada para a conicidade (C) de E. grandis aos 21 anos de idade.

Table 3. Summary of the variance analysis made for log taper (C) of a 21 year-old grown E. grandis.

\begin{tabular}{|c|c|c|c|}
\hline \multirow{2}{*}{ Causa de variação } & \multirow{2}{*}{ GL } & \multicolumn{2}{|c|}{ Quadrados médios } \\
\hline & & Tora 1 & Tora 2 \\
\hline Intensidade de desbaste (I) & 2 & $0,0032^{\text {n.s }}$ & $0,0071^{\mathrm{n} . \mathrm{s}}$ \\
\hline Adubo $(\mathrm{A})$ & 1 & $0,0013^{n . s}$ & $0,0007^{\text {n.s }}$ \\
\hline Classe (C) & 1 & $0,2094^{* *}$ & $0,0462 * *$ \\
\hline (I) $\mathrm{x}(\mathrm{A})$ & 2 & $0,0005^{\mathrm{n} . \mathrm{s}}$ & $0,0076^{\mathrm{n} . \mathrm{s}}$ \\
\hline (I) $\mathrm{x}(\mathrm{C})$ & 2 & $0,0035^{\mathrm{n} . \mathrm{s}}$ & $0,0083^{\text {n.s }}$ \\
\hline$(\mathrm{A}) \mathrm{x}(\mathrm{C})$ & 1 & $0,0013^{\mathrm{n} . \mathrm{s}}$ & $0,0004^{\mathrm{n} . \mathrm{s}}$ \\
\hline (I) $x(A) x(C)$ & 2 & $0,0008^{\mathrm{n} . \mathrm{s}}$ & $0,0038^{\mathrm{n} . \mathrm{s}}$ \\
\hline Resíduo & 36 & 0,0844 & 0,0687 \\
\hline $\mathrm{CV}_{\mathrm{e}}(\%)$ & & 21,03 & 22,86 \\
\hline Média $(\mathrm{cm} / \mathrm{m})$ & & 0,40 & 0,30 \\
\hline
\end{tabular}

Para o fator classe diamétrica, foi verificado que ocorreram diferenças significativas, tanto para a tora 1 quanto para a tora 2 . A $2^{\mathrm{a}}$ classe apresentou maior conicidade do que a $1^{\mathrm{a}}$ classe (Figura 2). Esse maior valor verificado na $2^{\text {a }}$ classe é devido a ela apresentar maiores diâmetros. Campos; Ribeiro (1983) também observaram que a conicidade aumenta com o aumento do DAP da árvore.

Scolforo; Figueiredo Filho (1995), em um estudo com algumas espécies de Eucalyptus, verificaram que, de forma geral, as espécies apresentaram fustes com boa forma, exceto o E. citriodora, que apresentou fuste de forma irregular, e o E. paniculata, que teve a forma do fuste prejudicada pela desrama incompleta e por cicatrizes. Em todas as espécies, a conicidade do tronco restringiu-se às três primeiras toras, evidenciando-se sua redução na segunda e terceira toras. Nessas toras foram obtidos maiores rendimentos no desdobro, principalmente para peças com grã paralela, com maior resistência mecânica. Na primeira tora, o valor da conicidade variou significativamente entre as espécies. Para a segunda tora, a conicidade foi menor para E. tereticornis e E. paniculata, e na terceira foi menor para $E$. pilularis e E. urophylla. Polli et al. (2006) verificaram em populações clonais de Eucalyptus, aos 55 meses de idade, que a conicidade, o achatamento e o encurvamento de toras não foram afetados 
significativamente pelos tratamentos de desrama artificial e não foram considerados defeitos na primeira tora de $3 \mathrm{~m}$.

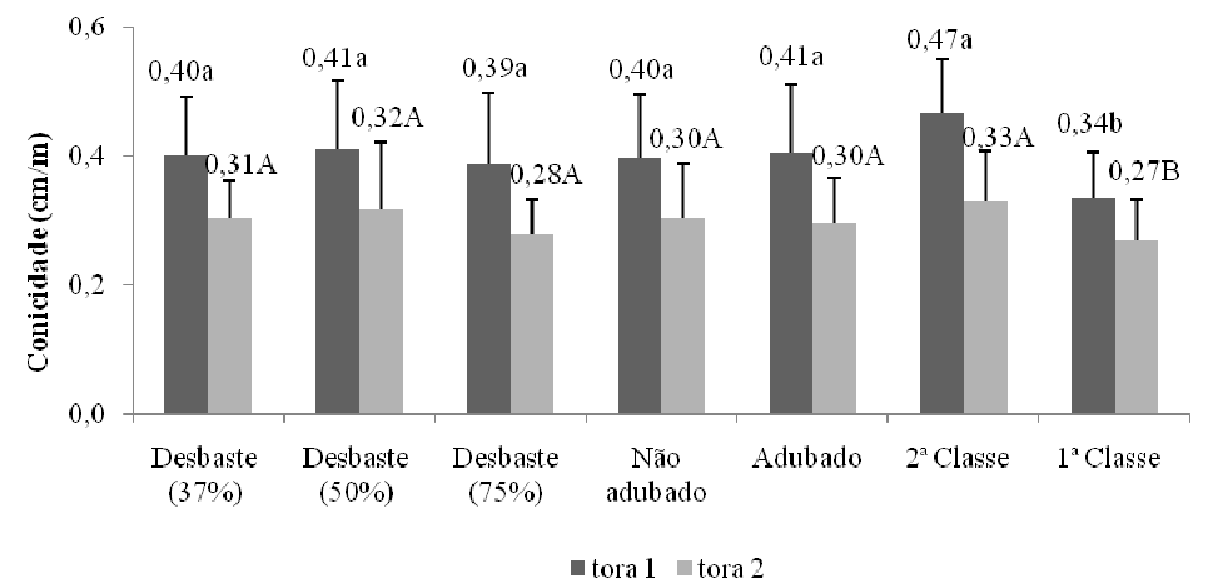

Nota: Médias seguidas da mesma letra não diferem entre si pelo teste Tukey ao nível de 5\% de significância.

Figura 2. Conicidade de toras (C) de E. grandis aos 21 anos de idade.

Figure 2. Log taper (C) of 21 year-old grown E. grandis.

De modo geral, foi observado que as toras apresentaram valores menores que $1 \mathrm{~cm} / \mathrm{m}$ de conicidade, o que não acarreta grandes perdas no aproveitamento da madeira (Figura 2). Esses baixos valores obtidos podem ser explicados pelo fato de não ter sido utilizada a tora basal, como já citado anteriormente neste trabalho. Oliveira (1997) encontrou valor médio para conicidade de toras de E. grandis, aos 16 anos, igual a $1,20 \mathrm{~cm} / \mathrm{m}$. Scanavaca Júnior (2001) também obteve valores menores que $1 \mathrm{~cm} / \mathrm{m}$ para E. urophylla e, assim, concluiu que a conicidade não influenciou no rendimento em madeira serrada. Barchet (2001) encontrou valores entre 0,55 a $1,36 \mathrm{~cm} / \mathrm{m}$ para diversas espécies de eucaliptos, enquanto que Haselein et al. (2004), para E. grandis de 27 anos, observaram valores médios de 1,41 cm/m.

$\mathrm{Na}$ tabela 4 e figura 1 observa-se que as toras 1 apresentaram valores de porcentagem de casca maiores significativamente do que as toras 2. Isso ocorreu devido às toras 1 terem maiores diâmetros. Essa mesma tendência foi observada por Paula Neto; Nunes (1992), Lopes (2000) e Santos et al. (2004).

Tabela 4. Teste t pareado para a conicidade (C) e porcentagem de casca (PC), para comparação efetuada entre as primeiras e as segundas toras de $E$. grandis aos 21 anos de idade.

Table 4. Test $\mathrm{t}$ for Log taper (C) and Percentage of bark (PC) for comparison between the first and second logs of 21 year-old grown E. grandis.

\begin{tabular}{lcccc}
\hline Característica & Tora 1 & Tora 2 & Teste t & Probabilidade t \\
\hline Conicidade $(\mathrm{cm} / \mathrm{m})$ & 0,40 & 0,30 & 5,51 & 0,0001 \\
Porcentagem de casca $(\%)$ & 7,95 & 6,66 & 4,01 & 0,0001 \\
\hline
\end{tabular}

As toras 1 também apresentaram maiores conicidades em relação às toras 2 (Tabela $4 \mathrm{e}$ Figura 2). A explicação desse resultado é devido aos maiores diâmetros observados nas toras 1 . Resultado semelhante também foi verificado por Scolforo; Figueiredo Filho (1995).

\section{CONCLUSÕES}

- A porcentagem de casca da tora 1 retirada entre 1,7 e 4,7 m de altura do fuste de Eucalyptus grandis é influenciada pelo desbaste e adubação e não sofre influência da classe de diâmetro. Essa variável decresce significativamente no intervalo de 1,7 a $9,4 \mathrm{~m}$ de altura do fuste.

- A conicidade varia com a classe de diâmetro, sendo mais acentuada na classe de maior diâmetro e ainda decresce da base para topo. 


\section{REFERÊNCIAS}

BARCHET, V. G. Potencialidade de espécie de Eucalyptus para produção de madeira serrada. 166 f. Dissertação (Mestrado em Ciências e Tecnologia de Madeira) - Escola Superior de Agricultura Luiz de Queiroz, Universidade de São Paulo, Piracicaba, 2001.

CALORI, J. V.; KIKUTI, P. Propriedades físicas e mecânicas da madeira de Eucalyptus dunnii aos 20 anos de idade. In: CONFERÊNCIA IUFRO SOBRE SILVICULTURA E MELHORAMENTO DE EUCALIPTOS, 1997. Salvador. Anais... Salvador: EMBRAPA/IPEF, 1997. p. 321 - 326.

CAMPOS, J. C. C.; RIBEIRO, J. C. Produção dos povoamentos de Eucalyptus spp. na região do Triângulo Mineiro. Boletim Técnico SIF, Viçosa, n. 1, 1983. 43 p.

COUTO, L.; BRANDI, R. M.; CONDE, A. R.; PAULA, F. Influência do espaçamento no crescimento do Eucalyptus urophylla, de origem híbrida, cultivado na região de Coronel Fabriciano, MG. Revista Árvore, Viçosa, v. 1, n. 2, p. 57 - 71, 1977.

CRÊSPO, E. A. Tensão de crescimento e suas consequências, controláveis e não controláveis, no desdobro e secagem do Eucalyptus grandis e Eucalyptus saligna. Muell. 119 f. Dissertação (Mestrado em Ciências e Tecnologia de Madeira) - Escola Superior de Agricultura Luiz de Queiroz, Universidade de São Paulo, Piracicaba, 2000.

DANIEL, T. W.; HELMS, J. A.; BACKER, F. S. Principles of silviculture. New York: McGraw-Hill, 1979. $492 \mathrm{p}$.

GARCIA, J. N. Gains end losses an sawn wood yield and quality thorough forest improvement, management and sawing strategies. In: INTERNATIONAL SYMPOSIUM EUCALYPTUS PLANTATIONS, Guangzhou, 2002. Proceedings... Guangzhou: WORLD SCIENTIFIC, 2002, p. 292 403.

GROSSER, D. Defeitos da madeira. Série Técnica FUPEF, Curitiba, n. 2, p. 1 - 62, 1980.

HASELEIN, C. R.; LOPES, M. C.; SANTINI, E. J.; LONGHI, S. J.; ROSO, S.; FERNANDES, D. L. G.; MENEZES, L. F. Características tecnológicas da madeira de árvores matrizes de Eucalyptus grandis. Ciência Florestal, Santa Maria, v. 14, n. 2, p. 145 - 155, 2004.

LIMA, I. L. Influência do desbaste e da adubação na qualidade da madeira serrada de Eucalyptus grandis Hill ex-Maiden. 137 p. Tese (Doutorado) - Escola Superior de Agricultura Luiz de Queiroz, Universidade de São Paulo, Piracicaba, 2005.

LIMA, I. L.; GARCIA, J. N.; STAPE, J. L. Influência do desbaste e da fertilização no deslocamento da medula e rachaduras de extremidade de tora de Eucalyptus grandis Hill ex-Maiden. Cerne, Lavras, v. 13, n. 2, p. 170 - 177, 2007.

LOPES, G. A. Qualidade da madeira de Eucalyptus saligna Smith, de Itatinga, associada aos padrões de casca apresentados pela população. $74 \mathrm{f}$. Dissertação (Mestrado em Ciências e Tecnologia de Madeira) - Escola Superior de Agricultura Luiz de Queiroz, Universidade de São Paulo, Piracicaba, 2000.

OLIVEIRA, J. T. S. Caracterização da madeira de eucalipto para a construção civil. $426 \mathrm{f}$. Tese (Doutorado em Engenharia Civil) - Escola Politécnica, Universidade de São Paulo, São Paulo, 1997.

OLIVEIRA, J. T. S.; HELLMEISTER, J. C.; SIMÕES, J. W.; TOMAZELLO FILHO, M. Caracterização da madeira de sete espécies de eucaliptos para a construção civil: 1- avaliações dendrométricas das árvores. Scientia Forestalis, Piracicaba, n. 56, p. 113 - 124, 1999.

PAULA NETO, F.; NUNES, J. R. S. Equações de volume de casca de Eucalyptus, de diferentes idades e condições de local, espécie e métodos de regeneração. Revista Árvore, Viçosa, v. 16, n. 2, p. 157 - 169, 1992. 
POLLI, H. Q.; REIS, G. G.; REIS, M. G. F.; VITAL, B. R.; PEZZOPANE, J. E. M.; FONTAN, I. C. I. Qualidade da madeira em clone de Eucalyptus grandis W. Hill ex-Maiden submetido a desrama artificial Revista Árvore, Viçosa, v. 30, n. 4, p. 557 - 566, 2006.

S.A.S. Institute Inc. SAS Procedures Guide. Version 8 (TSMO). SAS Institute Inc. Cary, N.C., 27513, USA, 1999.

SANTOS, P. E. T.; GARCIA, J. N.; GERALDI, I. O. Posição da tora na árvore e sua relação com qualidade da madeira serrada de Eucalyptus grandis. Scientia Forestalis, Piracicaba, n. 66, p. 142 - 151, 2004.

SCANAVACA JÚNIOR, L. Caracterização silvicultural, botânica e tecnológica do Eucalyptus urophylla $\mathbf{S}$. T. Blake e de seu potencial para utilização em serraria. $108 \mathrm{f}$. Dissertação (Mestrado em Ciências e Tecnologia de Madeira) - Escola Superior de Agricultura Luiz de Queiroz, Universidade de São Paulo, Piracicaba, 2001.

SCOLFORO, J. R. S.; FIGUEIREDO FILHO, A. Mensuração florestal - módulo 2: volumetria. Lavras: Esal, 1995. 126 p.

STANGER, T. K. A preliminary investigation of the variation in bark thickness, density and susceptibility to splitting in Eucalyptus dunnii Maiden. ICFR Bulletin series, Pietermaritzburg, n. 3, p. $12-16,1993$.

TOLFO, A. L. T.; PAULA, R. C.; BONINE, C. A. V.; BASSA, A.; VALLE, C. F. Parâmetros genéticos para caracteres de crescimento, de produção e tecnológicos na madeira de clones de Eucalyptus spp. Scientia Forestalis, Piracicaba, n. 67, p. 101 - 110, 2005. 